\title{
High-Spin Cobalt(II) Complex with Record-Breaking Anisotropy of the Magnetic Susceptibility According to Paramagnetic NMR Spectroscopy Data
}

\author{
Ya. A. Pankratova ${ }^{a, b}$, Yu. V. Nelyubina ${ }^{a}$, V. V. Novikov ${ }^{a}$, and A. A. Pavlov ${ }^{a, *}$ \\ ${ }^{a}$ Nesmeyanov Institute of Organoelement Compounds, Russian Academy of Sciences, Moscow, 119991 Russia \\ ${ }^{b}$ Moscow State University, Moscow, 119899 Russia \\ *e-mail: pavlov@ineos.ac.ru \\ Received April 11, 2020; revised May 14, 2020; accepted May 19, 2020
}

\begin{abstract}
The tetrahedral cobalt(II) complex $\left[\mathrm{CoL}_{2}\right]\left(\mathrm{HNEt}_{3}\right)_{2}$ (I), where L is 1,2-bis(methanesulfonamido)benzene, exhibiting the properties of a single-molecule magnet is synthesized and characterized. The electronic structure parameters of complex I are determined by paramagnetic NMR spectroscopy. They completely reproduce the results of less available methods of studying single-molecule magnets. The value of axial anisotropy of the magnetic susceptibility estimated for complex $\mathbf{I}\left(\Delta \chi_{\mathrm{ax}}=34.5 \times 10^{-32} \mathrm{~m}^{3}\right.$ at $\left.20^{\circ} \mathrm{C}\right)$ is record-breaking among all transition metal complexes studied by the NMR method, which provides wide possibilities for the use of complex I as a paramagnetic label for structural biology or as a contrast agent and even a temperature sensor for medical diagnostics. The data obtained indicate the advantages of paramagnetic NMR spectroscopy as a method of investigation of the magnetic properties and electronic structures of highly anisotropic transition metal complexes, which are precursors of many functional materials.
\end{abstract}

Keywords: cobalt(II) complexes, magnetic anisotropy, NMR spectroscopy, paramagnetic compounds, single-molecule magnets

DOI: $10.1134 / \mathrm{S} 1070328420120052$

\section{INTRODUCTION}

Paramagnetic metal complexes demonstrating high magnetic anisotropy have a number of promising practical applications in the areas of molecular electronics [1], magnetic resonance tomography (MRT) [2], and structural biology [3]. Owing to their properties, some of these complexes experienced the effect of an external permanent magnetic field can retain the magnetization gained in the field at the molecular level, i.e., can behave similarly to classical magnets [4, 5]. These compounds are commonly named singlemolecule magnets (SMM) and in future can form a basis for devices of information storage at the molecular level [6]. A similar behavior of SMM is explained by a significant magnetic anisotropy leading to the splitting of electron levels (so-called zero-field splitting). As a result, the magnetization relaxation is related to the surmounting of the energy barrier (1)

$$
\begin{gathered}
U=D S^{2}, \\
U=D\left(S^{2}-\frac{1}{4}\right),
\end{gathered}
$$

where $D$ is the splitting energy in the zero field, and $S$ is the total electron spin.
If the barrier $U$ is much higher than the thermal energy $k T$, the magnetization is retained for a long time in the case of the absence of side mechanisms (such as quantum tunneling, direct and Raman mechanisms) (2)

$$
\tau=\tau_{0} \exp \left(\frac{U}{k T}\right)
$$

where $\tau$ is the magnetization relaxation time.

A wide variety of methods for SMM studying is available at the moment, and the main of which is magnetometry. This method in the dynamic variation (in an alternating magnetic field) makes it possible to directly estimate magnetization relaxation times, and the simulation of magnetometry data in a permanent field allows one to study reasons for the observed properties in more detail by determining the electronic structure parameters ( $g$ tensor of zero-field splitting and others). However, the determination of these parameters implies the simulation of experimental data by some model that is not always unique, which can result in an excess parametrization of the model and, as a consequence, in violated results. A combination of various methods of studying electronic structures of paramagnetic metal complexes, such as optical spectroscopy [7], magnetic circular dichroism [8], 
or EPR spectroscopy [9], provides more reliable results $[10,11]$.

NMR spectroscopy traditionally used for the determination of structures of diamagnetic compounds is rarely applied for the investigation of paramagnetic coordination complexes because of difficulties in data detection and interpretation. However, in the recent years approaches of NMR spectroscopy for studying the magnetic properties and electronic struc- tures of these compounds are actively being developed by us [12] and other research groups [13-16].

In this work, we synthesized the tetrahedral cobalt(II) complex $\left[\mathrm{CoL}_{2}\right]\left(\mathrm{HNEt}_{3}\right)_{2}$ (I), where $\mathrm{L}$ is 1,2-bis(methanesulfonamido)benzene (Scheme 1), exhibiting the properties of SMM [17]. The complex was isolated in the pure state, and its composition and structure were unambiguously confirmed by the data of NMR spectroscopy and X-ray diffraction analysis.

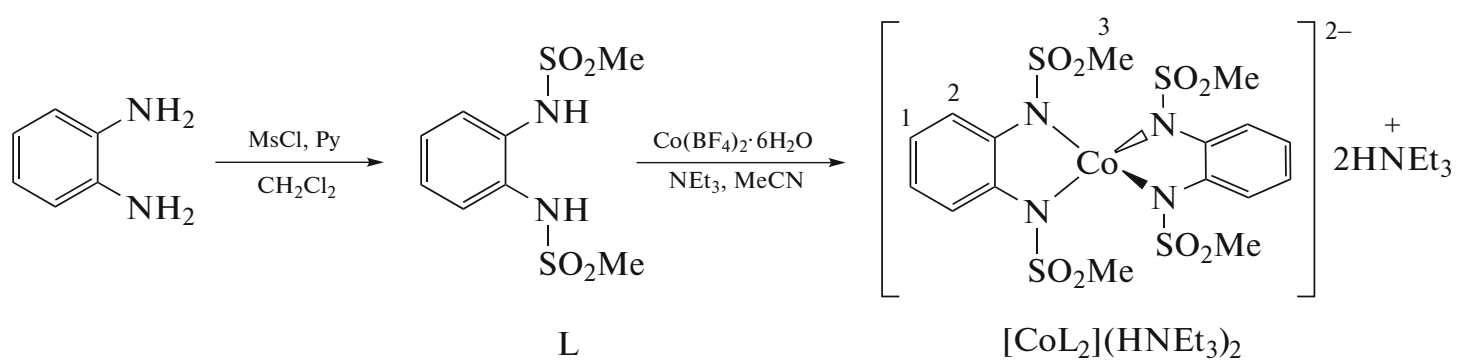

Scheme 1.

The parameters of the electronic structure of complex I were determined by paramagnetic NMR spectroscopy, and they are well consistent with the results obtained earlier by other investigation methods. The estimated axial anisotropy of magnetic susceptibility $\left(\Delta \chi_{\mathrm{ax}}=34.5 \times 10^{-32} \mathrm{~m}^{3}\right.$ at $\left.20^{\circ} \mathrm{C}\right)$ for complex I turned out to be record-breaking for all transition metal complexes studied by the NMR method. This provides wide possibilities for using this complex as a contrast agent or even a temperature sensor in MRT and as a paramagnetic label for the study of the spatial structures of biological macromolecules.

\section{EXPERIMENTAL}

Complex I and ligand L were synthesized in air using commercially available organic solvents and reagents. Elemental analyses to carbon, nitrogen, and hydrogen were carried out on a Carlo Erba microanalyzer (model 1106).

Synthesis of ligand $\mathbf{L}$ was carried out using the earlier published optimized procedure [18]. A solution of a mixture of pyridine $(0.4 \mathrm{~mL}, 5.55 \mathrm{mmol})$ and methanesulfonyl chloride $(0.3 \mathrm{~mL}, 4.07 \mathrm{mmol})$ was added dropwise to a boiling solution of $o$-phenylenediamine (200 $\mathrm{mg}, 1.85 \mathrm{mmol}$ ) in dichloromethane $(4 \mathrm{~mL})$. The reaction mixture was refluxed for $3.5 \mathrm{~h}$ and then slowly cooled down for $12 \mathrm{~h}$. The formed light pink precipitate was filtered off and washed with dichloromethane, and solvent residues were removed with an oil pump. The yield of the light pink finely crystalline product was $228 \mathrm{mg}$ (35\%).

${ }^{1} \mathrm{H}$ NMR $\left(\mathrm{CD}_{3} \mathrm{CN}, 300.15 \mathrm{MHz}, 18^{\circ} \mathrm{C}\right), \delta$, ppm: 2.98 (s, 6H, Me), $7.33(\mathrm{~m}, 2 \mathrm{H}, \mathrm{Ar}-\mathrm{H}), 7.49$ (m, 2H, Ar-H), 7.57 (br.s, 2H, NH).
Synthesis of $\left[\mathrm{CoL}_{2}\right]\left(\mathrm{HNEt}_{3}\right)_{2}$ (I) was carried out using the previously published optimized procedure [17]. Triethylamine $(130 \mu \mathrm{L}, 1.88 \mathrm{mmol})$ was added to a suspension of $\mathrm{L}(124 \mathrm{mg}, 0.47 \mathrm{mmol})$ in acetonitrile $(3 \mathrm{~mL})$. In $2 \mathrm{~min}$, a solution of $\left[\mathrm{Co}\left(\mathrm{BF}_{4}\right)\right] \cdot 6 \mathrm{H}_{2} \mathrm{O}$ $(80 \mathrm{mg}, 0.23 \mathrm{mmol})$ in acetonitrile $(0.5 \mathrm{~mL})$ was added to the obtained solution. The resulting violet solution was left overnight with vigorous stirring. After the end of the reaction, toluene $(4 \mathrm{~mL})$ was added to the reaction mixture, and the product was crystallized at reduced temperature $\left(4^{\circ} \mathrm{C}\right)$. The yield of the crimson-colored crystalline product was $45 \mathrm{mg}(26 \%)$.

${ }^{1} \mathrm{H}$ NMR $\left(\mathrm{CD}_{3} \mathrm{CN}, 600.22 \mathrm{MHz}, 18^{\circ} \mathrm{C}\right), \delta$, ppm: 112.37 (br.s, 4H, 2), 86.41 (br.s, 4H, 1), -7.10 (br.s, $\left.18 \mathrm{H}, \mathrm{CH}_{3}(\mathrm{Et})\right),-8.24$ (br.s, $\left.12 \mathrm{H}, \mathrm{CH}_{2}(\mathrm{Et})\right),-30.41$ (br.s, 12H, 3), -37.02 (br.s, 2H, NH).

For $\mathrm{C}_{28} \mathrm{H}_{52} \mathrm{~N}_{6} \mathrm{O}_{8} \mathrm{~S}_{4} \mathrm{Co}$
Anal. calcd., \%
C, 46.28
$\mathrm{H}, 6.65$
$\mathrm{N}, 10.67$
Found, \%
C, 46.24
$\mathrm{H}, 6.61$
$\mathrm{N}, 10.63$

NMR spectra were recorded for solutions in acetonitrile- $\mathrm{d}_{3}$ on Bruker Avance 300 and Bruker Avance 600 spectrometers (Larmor frequency for protons 300.15 and $600.22 \mathrm{MHz}$, respectively). Chemical shifts $(\delta, \mathrm{ppm})$ in NMR spectra were determined with respect to the residual signal of the solvent $\left({ }^{1} \mathrm{H}\right.$ NMR $1.94 \mathrm{ppm})$. The spectra of complex I were recorded using the following parameters: spectral range 300 ppm, detection time $0.5 \mathrm{~s}$, relaxation delay time $0.5 \mathrm{~s}$, pulse duration $6.5 \mathrm{~s}$, and acquisition number 32 . To increase the signal-to-noise ratio, the obtained free induction decays were processed by exponential weighing with the coefficient to $7 \mathrm{~Hz}$. 
Table 1. Selected crystallographic data and structure refinement parameters for $\left[\mathrm{CoL}_{2}\right]\left(\mathrm{HNEt}_{3}\right)_{2}$

\begin{tabular}{|c|c|}
\hline Parameter & Value \\
\hline$F W$ & 787.92 \\
\hline$T, \mathrm{~K}$ & 120 \\
\hline Crystal system & Orthorhombic \\
\hline Space group & $P 2_{1} 2_{1} 2_{1}$ \\
\hline$Z$ & 4 \\
\hline$a, \AA$ & $12.1858(6)$ \\
\hline$b, \AA$ & $16.0022(8)$ \\
\hline$c, \AA$ & $18.3286(9)$ \\
\hline$V, \AA^{3}$ & $3574.1(3)$ \\
\hline$\rho_{\text {calc }}, \mathrm{g} \mathrm{cm}^{-3}$ & 1.464 \\
\hline$\mu, \mathrm{cm}^{-1}$ & 7.69 \\
\hline$F(000)$ & 1668 \\
\hline $2 \theta_{\max }$, deg & 60 \\
\hline Number of measured reflections & 38231 \\
\hline Number of independent reflections $\left(R_{\text {int }}\right)$ & $10870(0.0740)$ \\
\hline Number of reflections with $I>2 \sigma(I)$ & 8726 \\
\hline Number of refined parameters & 434 \\
\hline$R_{1}, w R_{2}(I>2 \sigma(I))$ & $0.0439,0.0826$ \\
\hline$R_{1}, w R_{2}$ (all data) & $0.0623,0.0900$ \\
\hline GOOF & 0.997 \\
\hline$\Delta \rho_{\max } / \Delta \rho_{\min }$, e $\AA^{-3}$ & $0.411 /-0.339$ \\
\hline
\end{tabular}

X-ray diffraction analysis (XRD) of complex I was carried out on a Bruker APEX2 CCD diffractometer $\left(\mathrm{Mo} K_{\alpha}\right.$ radiation, graphite monochromator, $\omega$ scan mode). The structure was solved using the ShelXT program [19] and refined by full-matrix least squares using the Olex2 program [20] in the anisotropic approximation for $F_{h k l}^{2}$. The hydrogen atoms of the $\mathrm{NH}$ groups were localized from the difference Fourier electron density syntheses, and positions of other hydrogen atoms were calculated geometrically. The positions of all hydrogen atoms were refined in the isotropic approximation by the riding model. Selected crystallographic data and structure refinement parameters are presented in Table 1.

The atomic coordinates and other parameters of the structure of complex I were deposited with the Cambridge Crystallographic Data Centre (CIF file CCDC no. 1995722; http://www.ccdc.cam.ac.uk/).

The quantum-chemical calculations for complex I were performed using the ORCA, v. 4 program package [21] in terms of the density functional theory (DFT) [22]. The geometry of the complex was optimized without symmetry restraints using the PBE nonhybrid functional [23] in the def2-TZVP basis set [24]. The structure determined by the XRD method was used as the initial approximation. Solvation effects were taken into account in the framework of the CPCM model implemented in the ORCA, v. 4 pro- gram package. NMR spectra were recorded in an acetonitrile solution. The $g$ tensor and hyperfine coupling constants for protons were calculated on the basis of the obtained geometry of the complex using the PBE0 hybrid functional [25] in the def2-TZVP basis set [24]. Then the contact shift values were calculated using the following equation:

$$
\delta_{\text {con }}=\frac{A}{\hbar} \frac{g_{\text {iso }} \mu_{\mathrm{B}} S(S+1)}{3 \gamma_{\mathrm{I}} k T}
$$

where $g_{\text {iso }}$ is the isotropic electronic $g$ tensor, $\mu_{\mathrm{B}}$ is the Bohr magneton, $\hbar$ is Planck's constant, $\gamma_{\mathrm{I}}$ is the gyromagnetic ratio for proton, $k$ is the Boltzmann constant, $S$ is the spin quantum number equal to $3 / 2$ for the high-spin cobalt(II) complexes, and $T$ is temperature. The dimensionalities of all magnitudes are given in the SI system.

\section{RESULTS AND DISCUSSION}

The $\left[\mathrm{CoL}_{2}\right]\left(\mathrm{HNEt}_{3}\right)_{2}$ complex (I) was synthesized in a moderate yield using the earlier published optimized procedure [17] (Scheme 1) at room temperature. Attempts to crystallize the complex from the reaction mixture with diethyl ether resulted in the formation of a precipitate as a gray oil. However, the mixing of one fraction of the reaction mixture with one fraction of toluene followed by keeping the resulting 


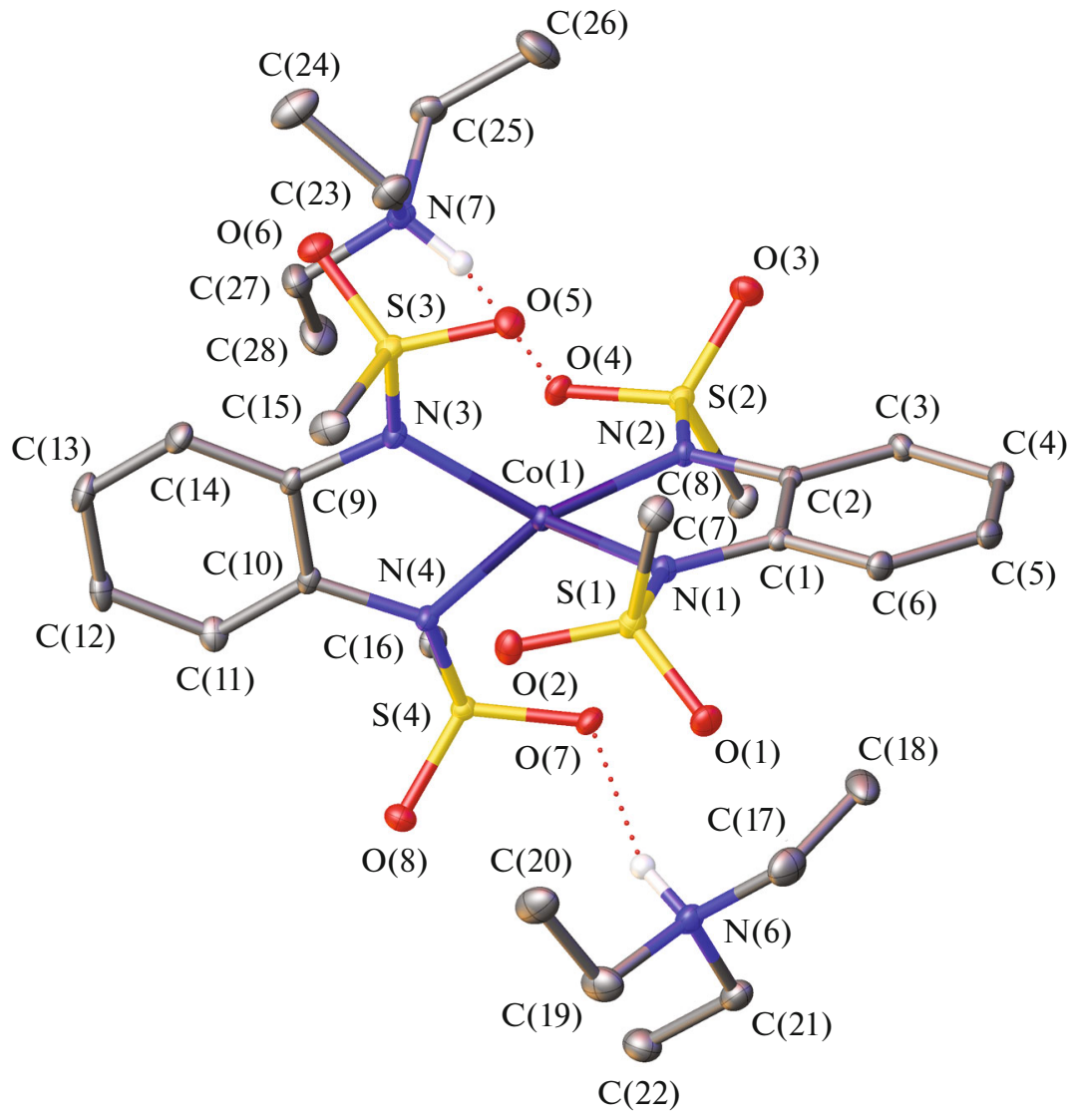

Fig. 1. General view of complex $\mathbf{I}$ in the representation of atoms by thermal vibration ellipsoids $(p=30 \%)$. Hydrogen atoms, except for those belonging to the NH groups, are omitted.

solution at $4{ }^{\circ} \mathrm{C}$ for $48 \mathrm{~h}$ afforded large crimson-like single crystals $(1-2 \mathrm{~mm})$ of complex $\mathbf{I}$, whose nature was unambiguously confirmed by the XRD results (Fig. 1). According to the data obtained, the molecular and crystal structures of complex I at $120 \mathrm{~K}$ (see Experimental) closely reproduce those determined previously [17] at $100 \mathrm{~K}$. In this structure, the anionic $\left[\mathrm{CoL}_{2}\right]^{2-}$ complex is bound to two $\mathrm{HNEt}_{3}^{+}$cations due to the N-H...O hydrogen bonds (N...O 2.829(4) and $2.725(4) \AA$, NHO $160.3(2)^{\circ}$ and $\left.160.7(2)^{\circ}\right)$ formed by the oxygen atoms of two $\mathrm{SO}_{2}$ groups. In complex I, the cobalt(II) ion exists in the high-spin state in the environment of four nitrogen atoms of two ligands $(\mathrm{Co}-\mathrm{N}$ 2.004(3)-2.018(3) ̊) forming an ideal tetrahedral environment around the ion, which can quantitatively be characterized by the so-called "continuous symmetry measures" [26] describing the deviation of the shape of the coordination polyhedron $\mathrm{CoN}_{4}$ from the ideal tetrahedron $\mathrm{S}(\mathrm{T}-4)$. The lower this value, the better the description of the polyhedron shape by this polyhedron. In the case of the studied complex $\mathbf{I}$, the corresponding value of $\mathrm{S}(\mathrm{T}-4)$ estimated from the $\mathrm{XRD}$ data at $120 \mathrm{~K}$ is 6.268 . For comparison, the devi- ation from the ideal planar square $\mathrm{S}(\mathrm{SP}-4)$ (so-called planar square "symmetry measures") reaches 23.219 , indicating the tetrahedral environment of the cobalt(II) ion in complex I.

NMR spectroscopy was used for the determination of the composition and structure of complex I in a solution and for the description of its magnetic properties and, correspondingly, its potential as a contrast agent in MRT or a paramagnetic label in structural biology. The Evans method is one of the traditional methods for studying paramagnetic compounds by NMR spectroscopy [27]. The method is based on the effect of the shift of the resonance position in the NMR spectrum of any nucleus in the solution of the paramagnetic substance. As a rule, the nuclei belonging to molecules of the solvent or reference compound specially added to the solution are chosen. The observed shift is proportional to the isotropic value of magnetic susceptibility $\left(\chi_{M}\right)$ of the paramagnetic complex under study

$$
\begin{aligned}
\chi_{M} & =\frac{\Delta \delta M}{v_{0} S_{\mathrm{f}} m}-\chi_{\mathrm{M}}^{\mathrm{dia}}, \\
\chi_{\text {iso }} & =\left(\chi_{\|}+2 \chi_{\perp}\right) / 3,
\end{aligned}
$$




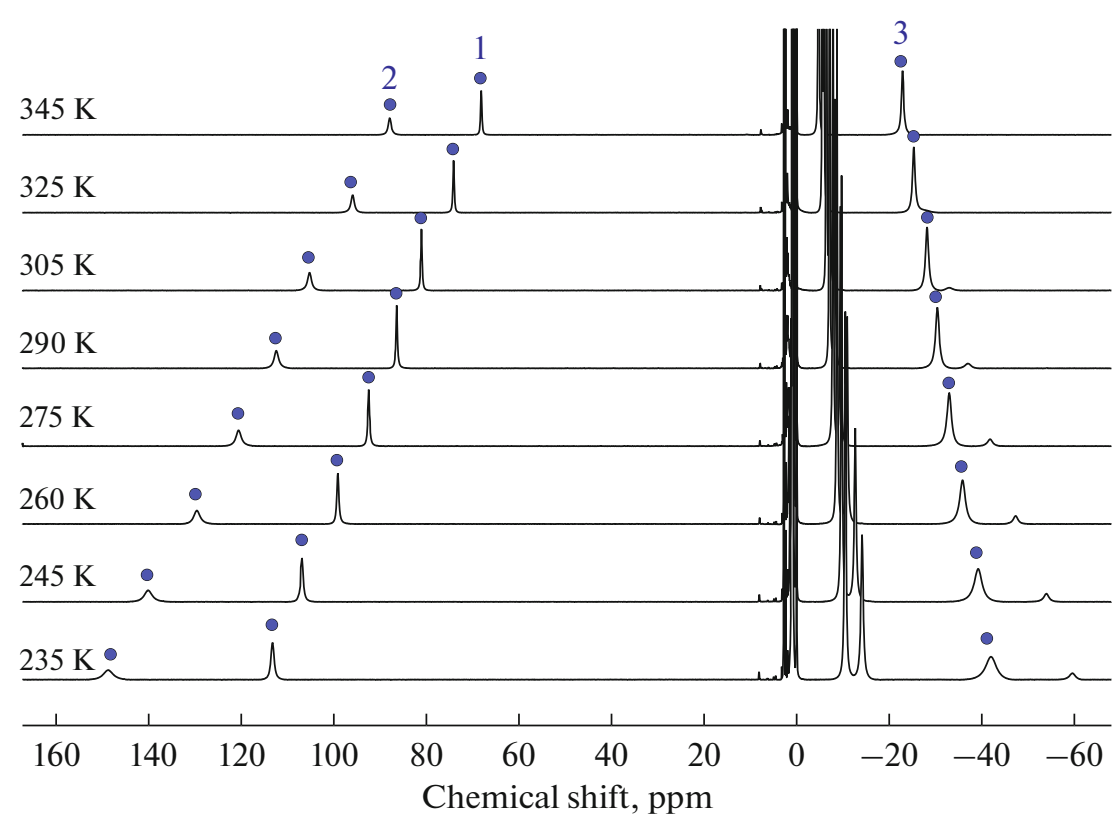

Fig. 2. ${ }^{1} \mathrm{H}$ NMR spectra of complex I $\left(600.22 \mathrm{MHz}, \mathrm{CD}_{3} \mathrm{CN}\right)$ in the temperature range $235-345 \mathrm{~K}$. The nuclei are enumerated in Scheme 1.

where $\chi_{\|}$and $\chi_{\perp}$ are the parallel and perpendicular components of the $\chi$ tensor, respectively.

At the same time, the nuclei of the paramagnetic complex demonstrate a higher shift in the NMR spectra. This shift is accepted to be named paramagnetic $\left(\delta_{\mathrm{par}}\right)$

$$
\delta_{\text {tot }}=\delta_{\text {dia }}+\delta_{\text {par }}=\delta_{\text {dia }}+\delta_{\mathrm{c}}+\delta_{\mathrm{pc}}
$$

where $\delta_{\text {tot }}$ and $\delta_{\text {dia }}$ are the observed chemical shift and its diamagnetic component caused by the shielding of paired electrons, respectively.

In turn, the $\delta_{\mathrm{par}}$ shift is usually divided into the contact shift $\left(\delta_{\mathrm{c}}\right)$ caused by the spin density on the nucleus and pseudocontact shift $\left(\delta_{\mathrm{pc}}\right)$. Since it is fairly difficult to experimentally determine the spin density distribution in the molecule, the contact shift is usually calculated by quantum-chemical methods. Since the pseudo-contact shift is based on the dipolar interaction of magnetic moments of the nucleus and unpaired electron, the value of the shift depends on the mutual arrangement of interacting particles. The paramagnetic ion is chosen as a localization center of unpaired electrons, and the nuclear coordinates are determined from the XRD data and/or quantum-chemical calculation results with the geometry optimization of the molecule.

$$
\delta_{\mathrm{pc}}=\frac{1}{12 \pi r^{3}}\left[\Delta \chi_{\mathrm{ax}}\left(3 \cos ^{2} \theta-1\right)\right],
$$

where $r$ and $\theta$ are the polar coordinates of the nucleus.

The axial anisotropy of the magnetic susceptibility expressed as a difference between the eigenvalues of the corresponding tensor $\left(\Delta \chi_{\mathrm{ax}}=\chi_{\|}-\chi_{\perp}\right)$ is an important characteristic of the electronic structure of the molecule. First, the value of anisotropy itself characterizes the prospects of the compound as a paramagnetic label and contrast agent or as a temperature sensor in MRT, since it seems reasonable to choose systems with the highest anisotropy for these purposes. Second, the anisotropy $\Delta \chi_{\mathrm{ax}}$ along with the isotropic value of $\chi_{\text {iso, }}$, which can be obtained by the Evans method, completely characterizes the magnetic susceptibility tensor $\chi$ directly related to the electron energy levels via the van Vleck equation

$$
\chi_{\mathrm{a}}=\frac{N_{A} k T}{10} \frac{\partial^{2}}{\partial B_{\mathrm{a}}^{2}} \ln \left(\sum_{i} e^{-\frac{\psi_{i}|\hat{\mathscr{E}}| \psi_{i}}{k T}}\right),
$$

where $a=\|$ or $\perp$ (parallel or perpendicular component of the tensor); and $\hat{\mathscr{H}}$ is the spin-Hamiltonian (operator of the energy of magnetic interactions) expressed by the following equation:

$$
\hat{\mathscr{H}}=\frac{D}{\hbar}\left(S_{z}^{2}-\frac{S^{2}}{3}\right)+\frac{\mu_{\mathrm{B}}}{\hbar} g B \hat{S},
$$

where $D$ is the splitting energy in the zero field.

The ${ }^{1} \mathrm{H}$ NMR spectra for the chosen cobalt complex I were detected in a range of 235-345 K (Fig. 2). The observed chemical shifts for nuclei 1 and 2 (the corresponding enumeration is presented in Scheme 1) were simulated by Eqs. (6) and (7). The NMR spectra for free ligand $\mathrm{L}$ were used as $\delta_{\mathrm{dia}}$, and $\delta_{\mathrm{c}}$ was calculated by the quantum-chemical methods (see Experimental). An excellent coincidence of the experimental shifts of nuclei 1 and 2 with the calculated data (Fig. 3) 


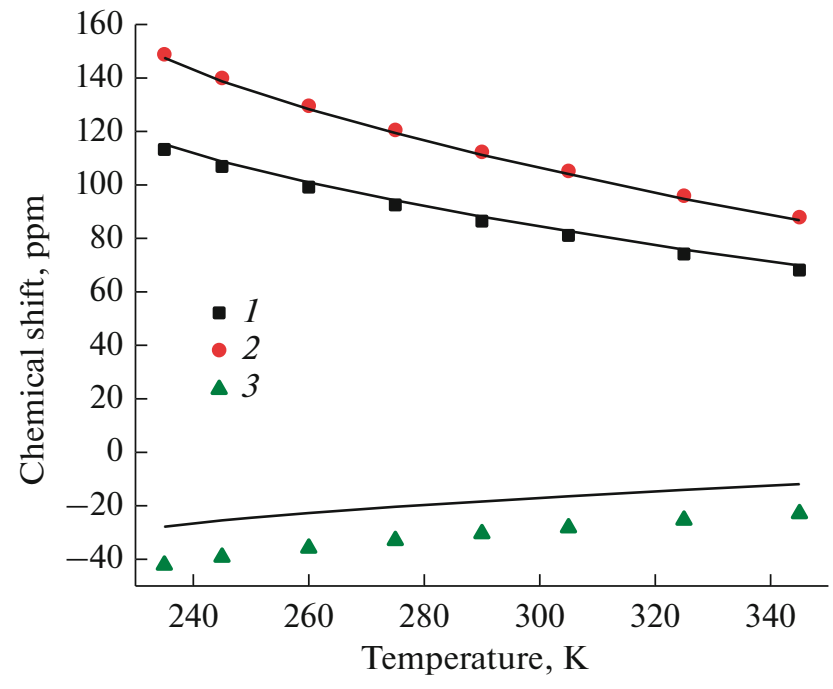

Fig. 3. Temperature dependences of the chemical shifts in the ${ }^{1} \mathrm{H}$ NMR spectra for nuclei $1-3$ of complex I $\left(600.22 \mathrm{MHz}, \mathrm{CD}_{3} \mathrm{CN}\right)$. The experimental values are shown by points, and the calculated values are shown by solid lines.

was achieved for all temperatures and characterized by the high criterion $R^{2}>0.99$, which made it possible to obtain reliable values for the magnetic susceptibility anisotropy $\left(\Delta \chi_{\mathrm{ax}}\right)$. The convergence of the experimental shifts with the calculated values for the protons of the methyl group was expectedly somewhat lower. A similar divergence discussed previously [28, 29] is associated with the conformational lability of coordination compounds in the solution. At room temperature, the corresponding value of $\Delta \chi_{\mathrm{ax}}$ for complex $\mathbf{I}$ is $34.5 \times 10^{-32} \mathrm{~m}^{3}$, which is the absolute record among the transition metal complexes studied by the NMR method. In particular, for the majority of the cobalt(II) complexes (cobalt(II) is the most anisotropic ion among transition metals), this value lies in a range of 3-7 $\times 10^{-32} \mathrm{~m}^{3}$ [30], whereas it reaches $20-$ $25 \times 10^{-32} \mathrm{~m}^{3}$ for the most anisotropic complexes known at the moment [31]. Such a high magnetic anisotropy allows one to consider complex $\mathbf{I}$ as a promising paramagnetic label for structural biology or a contrast agent and even a temperature sensor for MRT. This is due to the fact that a higher magnetic anisotropy of the paramagnetic compound leads to the appearance of stronger paramagnetic shifts in the NMR spectrum, which makes it possible to study larger macromolecules and more easily detect the MRT signal even at a low concentration of the agent and, in the case of a sensor, to determine the temperature in vivo more precisely. It should be mentioned that many magnetically anisotropic complexes are chemically unstable in the solution. However, when using NMR spectroscopy, the chemical stability of the complex in this phase state is confirmed de facto, which is an important advantage of the method.

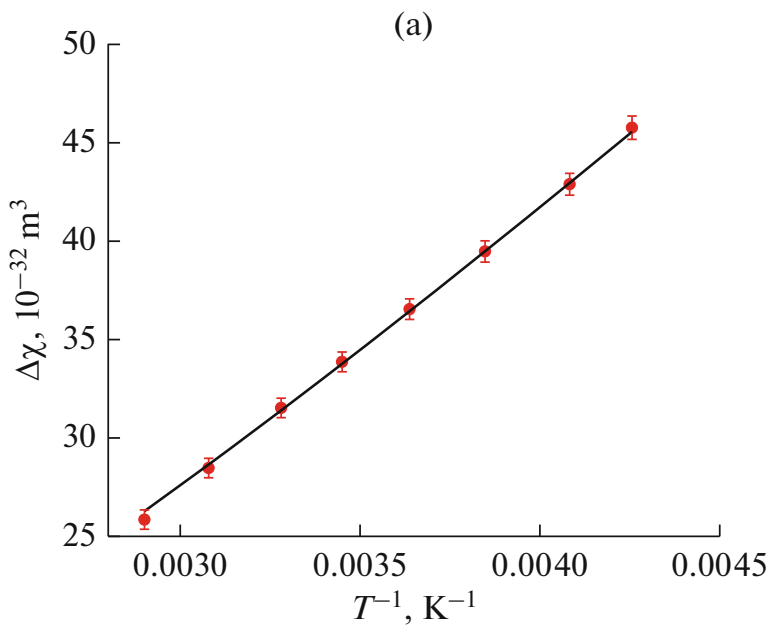

(b)

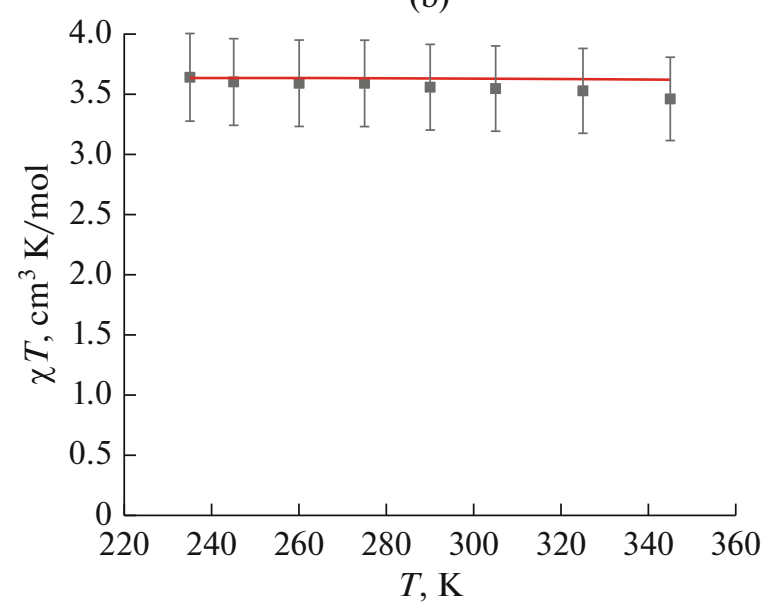

Fig. 4. Temperature dependences of (a) $\Delta \chi_{a x}$ and (b) $\chi_{\text {iso }}$ for complex I. The latter dependence was obtained by the Evans method. Solid lines correspond to the simultaneous simulation of the data by the spin-Hamiltonian with the parameters $g_{\perp}=2.49, g_{\|}=3.13$, and $D=-140 \mathrm{~cm}^{-1}$.

The application of the Evans method made it possible to determine the isotropic value of the magnetic susceptibility $\left(\chi_{\text {iso }}\right)$ for complex $\mathbf{I}$ in a range of 235$345 \mathrm{~K}$. The data obtained were simulated by the van Vleck equation (8) together with the temperature dependence of the magnetic susceptibility anisotropy $\Delta \chi_{\mathrm{ax}}$ using the spin-Hamiltonian (9), which gave the following parameters of the electronic structure: $g_{\perp}=$ $2.49 \pm 0.07, g_{\|}=3.13 \pm 0.10$, and $D=-140 \pm 30 \mathrm{~cm}^{-1}$ (Fig. 4). The indicated scatter of the values is due to both an insufficiently high accuracy of the quantumchemical calculation of the contact shifts and a measurement inaccuracy of the Evans method [32, 33]. The obtained values are consistent with the energy of the second Kramers doublet $\left(230 \mathrm{~cm}^{-1}\right)$ estimated from the data of far-IR spectroscopy in the magnetic field [17] corresponding to the splitting energy in the zero field $|D|=115 \mathrm{~cm}^{-1}$. 
Thus, we synthesized the tetrahedral cobalt(II) complex (I) with the properties of SMM. The structure of complex I was confirmed by the XRD method, and its magnetic properties in solutions were studied by paramagnetic NMR spectroscopy. In particular, $\Delta \chi_{\text {ax }}$ was $34.5 \times 10^{-32} \mathrm{~m}^{3}$ at room temperature, which is record-breaking among all known transition metal complexes studied by the NMR method. This makes it possible to consider complex $\mathbf{I}$ as a promising paramagnetic label for structural biology and a contrast agent and temperature sensor in MRT. The isotropic magnetic susceptibility measured in parallel by the Evans method along with the magnetic susceptibility anisotropy estimated during an analysis of the observed chemical shifts in the NMR spectra were simulated by the van Vleck equation in terms of the spin-Hamiltonian formalism. The parameters obtained for the electronic structure $\left(g_{\perp}=2.49 \pm 0.07\right.$, $g_{\|}=3.13 \pm 0.10$, and $D=-140 \pm 30 \mathrm{~cm}^{-1}$ ) are completely consistent with the data by less available methods of investigation of SMM, such as far-IR spectroscopy in the magnetic field and magnetic circular dichroism. The results obtained also demonstrate that paramagnetic NMR spectroscopy is promising as a method for studying the magnetic properties and electronic structures of highly anisotropic transition metal complexes, which are precursors of many functional materials.

\section{ACKNOWLEDGMENTS}

The XRD studies were supported by the Ministry of Science and Higher Education of the Russian Federation using the scientific equipment of the Center of Study of Structure of Molecules at the Nesmeyanov Institute of Organoelement Compounds (Russian Academy of Sciences).

\section{FUNDING}

This work was supported by the Russian Science Foundation, project no. 18-73-00113.

\section{CONFLICT OF INTEREST}

The authors declare that they have no conflict of interest.

\section{OPEN ACCESS}

This article is distributed under the terms of the Creative Commons Attribution 4.0 International License (http:// creativecommons.org/licenses/by/4.0/), which permits unrestricted use, distribution, and reproduction in any medium, provided you give appropriate credit to the original author(s) and the source, provide a link to the Creative Commons license, and indicate if changes were made.

\section{REFERENCES}

1. Winpenny, R.E., Ang. Chem. Int. Ed., 2008, vol. 47, no. 42 , p. 7992.
2. Sinharay, S. and Pagel, M.D., Ann. Rev. Anal. Chem., 2016, vol. 9, p. 95.

3. Yagi, H., Pilla, K.B., Maleckis, A., et al., Structure, 2013, vol. 21, no. 6, p. 883.

4. Sessoli, R., Gatteschi, D., Caneschi, A., et al., Nature, 1993, vol. 365, no. 6442, p. 141.

5. Sessoli, R. and Powell, A.K., Coord. Chem. Rev., 2009, vol. 253, nos. 19-20, p. 2328.

6. Frost, J.M., Harriman, K.L., and Murugesu, M., Chem. Sci, 2016, vol. 7, no. 4, p. 2470.

7. Chia, Y. and Tay, M., Dalton Trans., 2014, vol. 43, no. 35 , p. 13159.

8. Larrabee, J.A., Alessi, C.M., Asiedu, E., et al., J. Am. Chem. Soc., 1997, vol. 119, no. 18, p. 4182.

9. Palacios, M.A., Nehrkorn, J., Suturina, E.A., et al., Chem.-Eur. J., 2017, vol. 23, no. 48, p. 11649.

10. Ishikawa, N., J. Phys. Chem. A, 2003, vol. 107, no. 30, p. 5831.

11. Pavlov, A.A., Nehrkorn, J., Pankratova, Y.A., et al., Phys. Chem. Chem. Phys., 2019, vol. 21, no. 16, p. 8201.

12. Novikov, V.V., Pavlov, A.A., Nelyubina, Y.V., et al., J. Am. Chem. Soc., 2015, vol. 137, no. 31, p. 9792.

13. Polovkova, M.A., Martynov, A.G., Birin, K.P., et al., Inorg. Chem., 2016, vol. 55, no. 18, p. 9258.

14. Damjanovic, M., Katoh, K., Yamashita, M., and Enders, M., J. Am. Chem. Soc., 2013, vol. 135, no. 38, p. 14349.

15. Hiller, M., Krieg, S., Ishikawa, N., and Enders, M., Inorg. Chem., 2017, vol. 56, no. 24, p. 15285.

16. Suturina, E.A., Mason, K., Botta, M., et al., Dalton Trans., 2019, vol. 48, no. 23, p. 8400.

17. Rechkemmer, Y., Breitgoff, F.D., Van Der Meer, M., et al., Nat. Commun., 2016, vol. 7, no. 1, p. 1.

18. Wu, Z., Wen, K., Zhang, J., and Zhang, W., Org. Lett., 2017 , vol. 19 , no. 11 , p. 2813.

19. Sheldrick, G.M., Acta Crystallogr., Sect. A: Found. Crystallogr., 2008, vol. 64, p. 112.

20. Dolomanov, O.V., Bourhis, L.J., Gildea, R.J., et al., J. Appl. Crystallogr., 2009, vol. 42, p. 339.

21. Neese, F., WIREs Comput. Mol. Sci., 2018, vol. 8, no. 1.

22. Runge, E. and Gross, E.K.U., Phys. Rev. Lett., 1984, vol. 52, no. 12, p. 997.

23. Perdew, J.P., Burke, K., and Ernzerhof, M., Phys. Rev. Lett., 1996, vol. 77, no. 18, p. 3865.

24. Weigend, F. and Ahlrichs, R., Phys. Chem. Chem. Phys., 2005 , vol. 7 , no. 18 , p. 3297.

25. Adamo, C. and Barone, V., J. Chem. Phys., 1999, vol. 110 , no. 13, p. 6158 .

26. Alvarez, S., Chem. Rev., 2015, vol. 115, no. 24, p. 13447.

27. Schubert, E.M., J. Chem. Educ., 1992, vol. 69, no. 1, p. 62.

28. Comba, P., Enders, M., Großhauser, M., et al., Dalton Trans., 2017, vol. 46, no. 1, p. 138.

29. Damjanovič, M., Samuel, P.P., Roesky, H.W., and Enders, M., Dalton Trans., 2017, vol. 46, no. 16, p. 5159.

30. Bertini, I., Luchinat, C., Parigi, G., and Pierattelli, R., ChemBioChem., 2005, vol. 6, no. 9, p. 1536.

31. Pavlov, A.A., Savkina, S.A., Belov, A.S., et al., ACS Omega, 2018, vol. 3, no. 5, p. 4941.

32. Ostfeld, D. and Cohen, I.A., J. Chem. Educ., 1972, vol. 49, no. 12, p. 829.

33. Yatsunyk, L.A. and Walker, F.A., Inorg. Chem., 2004, vol. 43, no. 2, p. 757.

Translated by E. Yablonskaya 